\title{
Comparativo sintomático sobre a covid-19 e dengue
}

\section{Symptomatic comparison on covid-19 and dengue}

DOI: $10.46919 / \operatorname{archv2n3-029}$

Recebimento dos originais: 01/01/2021

Aceitação para publicação: 31/03/2021

\author{
Alda Andressa Moreira Luna \\ Instituição: Universidade Brasil \\ Endereço: Rua Euripedes Teixeira de Resende, №757 \\ Parque Universitário - Fernandópolis - São Paulo CEP- 15601-318 \\ E-mail: thomasfmeira@gmail.com \\ Thomás Fernandes Meira Andrade \\ Instituição: Universidade Brasil \\ Endereço: Rua Euripedes Teixeira de Resende, No757 \\ Parque Universitário - Fernandópolis - São Paulo CEP- 15601-318 \\ E-mail: thomasfmeira@gmail.com \\ Dr. Márcio César Reino Gaggini \\ Mestrado em Bioengenharia pela UNICASTELO \\ Instituição: Universidade Brasil \\ Endereço: Rua Euripedes Teixeira de Resende, $N^{\circ} 757$ \\ Parque Universitário - Fernandópolis - São Paulo CEP- 15601-318 \\ E-mail: thomasfmeira@gmail.com
}

\section{RESUMO}

O Brasil enfrenta epidemias de dengue desde 1986 sazonalmente de março a junho e agora associado com a pandemia do covid-19. O impacto da COVID-19 no sistema público de saúde é perceptível pela elevada demanda por internação hospitalar, levando ao esgotamento da oferta de leitos de terapia intensiva e ventiladores pulmonares em algumas regiões do país. Provocado impactos em todos os setores da sociedade, principalmente nos sistemas de saúde, devido à sua rápida disseminação por todos os continentes, sua capacidade de provocar mortes em populações vulneráveis, além do insuficiente conhecimento científico sobre o vírus, patogenia e tratamento.E grande relevância relacionar os sintomas de pacientes com Covid-19 apresentados no momento do acolhimento dos usuários do SUS durante o atendimento na ESF e relacionar com os sintomas apresentados pela Dengue registrados pela Cartilha do Ministério da Saúde. Para tanto utilizou-se coleta retrospectivos dos dados dos pacientes já confirmados com o teste positivo para Covid-19, após esses dados foram utilizados os prontuários eletrônico dos pacientes e considerado quais as principais queixas na primeira consulta. O Covid- 19 e a dengue há diversos sintomas similares podendo ser confundido quando apresentados isoladamente ou até mesmo em conjunto a depender dos sintomas, dentre os sintomas frequentes em ambas as doenças estão febre, mialgia, cefaleia, dor abdominal, diarreia, diante desse fato e necessário sempre suspeita de ambas as doenças até sua adequada investigação, e assim estipular adequado tratamento. Assim como na dengue o covid- 19 e mais graves em pessoas com a presença de comorbidades e idosos.

Ressaltando que o covid- 19 e uma doença nova e que ainda a muita a ser descoberto, e para tanto e de suma importância à continua monitorização dos sintomas que surgem com o avançar das doenças.

Palavras-chave: Covid-19. Dengue. Manifestações clinicas. 


\section{ABSTRACT}

Brazil has faced dengue epidemics since 1986 seasonally from March to June and now associated with the covid-19 pandemic. The impact of covid-19 on the public health system is noticeable by the high demand for hospital admission, leading to depletion of the supply of intensive care beds and lung ventilators in some regions of the country. It has caused impacts in all sectors of society, especially in health systems, due to its rapid spread across all continents, its ability to cause deaths in vulnerable populations, in addition to insufficient scientific knowledge about the virus, pathogenesis and treatment.It is of great relevance to relate the symptoms of patients with Covid-19 presented at the time of reception of users of the SUS during care in the ESF and relate them to the symptoms presented by Dengue recorded by the Booklet of the Ministry of Health. For this, retrospective data collection of patients already confirmed positive for Covid-19 was used, after these data were used the electronic medical records of patients and considered what the main complaints in the first consultation. Covid-19 and dengue have several similar symptoms and can be confused when presented alone or even together depending on the symptoms, among the frequent symptoms in both diseases are fever, myalgia, headache, abdominal pain, diarrhea, before this fact and it is necessary to always suspect both diseases until their proper investigation, and thus stipulate appropriate treatment. As with dengue, covid-19 is more severe in people with the presence of comorbidities and the elderly.

Covid-19 is a new disease and there is still much to be discovered, so it is of utmost importance to continue to monitor the symptoms that arise as the disease progresses.

Keywords: Covid-19. Dengue fever. Clinical manifestations.

\section{INTRODUÇÃO}

O Brasil enfrenta epidemias de dengue desde 1986 sazonalmente de março a junho e agora associado com a pandemia do covid-19. São doenças de causa viral que apresentam diferentes agentes etiológicos e diferentes meios de transmissão. A Dengue é causada pelo vírus DENGV transmitida mosquito vetor (Aedes aegypti), (FIOCRUZ, 2020). A COVID-19, doença emergente causada pelo novo coronavírus denominado SARS-CoV-2 transmitido através de gotículas e aerossóis de pessoas infectadas.

A elevação no nível pluviométrico e as falhas nas ações de controle do mosquito vetor (Aedes aegypti) contribuem para o aumento de casos de dengue nesse período (GUZMAN 2015) e as aglomerações, uso inadequado de equipamentos de proteção individual, não uso de máscara, a não lavagem ou lavagem incorreta das mãos entre outros fatores contribuem para a propagação do vírus SARS-CoV-2. (BRASIL,2020)

O impacto da COVID-19 no sistema público de saúde é perceptível pela elevada demanda por internação hospitalar, levando ao esgotamento da oferta de leitos de terapia intensiva e ventiladores pulmonares em algumas regiões do país. (Barreto ML 2020). COVID-19 e dengue apresentam semelhanças clínicas e laboratoriais. (Yan G, 2020) observaram que pacientes infectados pelo SARS-CoV-2 e diagnosticados com dengue, por meio de teste rápido, evoluíram para quadros clínicos mais graves, retardando o tratamento eficaz. Diagnósticos falsos positivos e métodos laboratoriais menos sensíveis podem gerar, não somente, complicações na saúde do paciente, como favorecem a expansão da COVID- 
19, sobrecarregando os sistemas de saúde pública. Provocado impactos em todos os setores da sociedade, principalmente nos sistemas de saúde, devido à sua rápida disseminação por todos os continentes, sua capacidade de provocar mortes em populações vulneráveis, além do insuficiente conhecimento científico sobre o vírus, patogenia e tratamento.

A conscientização dos profissionais para a importância de notificação dos casos é de grande importância para evitar que a epidemia seja "subdimensionada" de dengue simultaneamente à ocorrência de casos de COVID-19.

Para enfrentar bem essas doenças é necessário que a população utilize de estratégias eficazes e conscientização pessoal e social, no sentido de eliminar criadouros de mosquitos, lavagem das mãos, uso de máscaras e, principalmente, distanciamento social. Além disso, é fundamental fortalecer o SUS e direcionar recursos para organizar os serviços de saúde, oferecer a toda a população um atendimento de qualidade tanto na atenção primária à saúde, onde são atendidos os casos leves de ambas as doenças, como na assistência hospitalar direcionada aos casos de maior complexidade. Por ser concebida como acesso preferencial no atendimento de pessoas com suspeita de dengue e COVID-19, a atenção primária à saúde é um componente fundamental do sistema de saúde, devendo ser fortalecida e estar preparada para o atendimento oportuno, manejo clínico adequado e referenciamento dos casos graves, bem como atenção integral às condições sensíveis neste nível de atendimento

As medidas adotadas pelos gestores para minimizar os danos da pandemia, como o lockdown e demais restrições tem dificultado o acesso de profissionais da área da saúde a terem acesso a essas casas, exemplo os agentes comunitários de saúde que ficaram limitados ao exercer seu papel de controle ao mosquito vetor da dengue.

Por não possuírem tratamento específicos e achados patognomônicos essas doenças são tratadas com sintomáticos de acordo com a repercussão orgânica de cada indivíduo acometido e sua gravidade. E para diagnosticar ou afirmar que um paciente está infectado com Dengue ou Covid-19 necessita de exames laboratoriais e os testes diagnósticos possuem momentos específicos da infecção para obter melhores resultados.

Realizando uma boa avaliação clínica, direcionando a anamnese em coletar os dados de tempo de início de sintomas, relacionar os sintomas mais específicos, solicitar os testes no tempo correto, aumenta a eficácia no tratamento.

\section{OBJETIVOS}

O objetivo desse estudo é relacionar os sintomas de pacientes com Covid-19 apresentados no momento do acolhimento dos usuários do SUS durante o atendimento na ESF e relacionar com os sintomas apresentados pela Dengue registrados pela Cartilha do Ministério da Saúde. 


\section{MÉTODOS}

Para a realização desse artigo foram coletados os dados retrospectivos dos pacientes já confirmados com o teste positivo para Covid-19, após esses dados foram utilizados os prontuários eletrônico dos pacientes onde anotamos quais as principais queixas na primeira consulta. Após comparamos esses dados com os sintomas da Dengue que estão descritos na cartilha do ministério da Saúde.

\section{DESENVOLVIMENTO}

Em dezembro de 2019 começou a ser notícia que algumas pessoas que residiam na cidade de Wuhan localizado na China, começou a apresentar uma pneumonia de causa desconhecida, no cenário epidemiológico descobriu uma ligação dessas pessoas com um mercado atacadista que comercializava espécies de animais vivos das mais variadas espécies.

Investigando mais esses pacientes descobriu que se tratava de um vírus novo da família Coronavidae, que tem relação com a Síndrome Respiratória Aguda Grave (SARS) e à Síndrome Respiratória do Oriente Médio (MERS) (SAAVEDRA-VELASCO et al., 2020). Esse vírus foi se alastrando pelo mundo e em marco de 2020 a Organização Mundial de Saúde (OMS), declarou a pandemia da doença causada pelo coronavírus (Cov) 2019, COVID-19, Síndrome Respiratória Aguda Grave CoV [SARS-CoV2], que logo impactou vários países causando muitas mortes.

No Brasil o primeiro caso confirmado foi de um homem de 61 anos com história de viagem à Itália, após essa notificação apresentou vários outros casos que se alastraram rapidamente no país, aumentando o número de casos e de óbitos (BRASIL, 2020). Paralelo a isso, o país continua a enfrentar várias doenças tropicais, como a dengue, e essa simultaneidade tem seus impactos (LORENZ et al., 2020). A evolução dessa doença é rápida chegando a estado grave em pouco tempo, sendo de extrema necessidade que os profissionais de saúde saibam reconhecer de maneira mais precoce possível os sinais de alarme e tomar as medidas efetivas cabíveis.

A Dengue no Brasil tem o agravo da sazonalidade que pode se estender entre janeiro e junho com pico geralmente em março aumentando a preocupação de coexistir a associação das duas doenças. Ademais, foi observado que a faixa etária acima de 60 anos concentra 60,4\% dos óbitos de dengue confirmados (BRASIL, 2020), e que a população idosa também concentra o número de casos de COVID-19 (BRASIL, 2020). Essas similaridades, além da clínica parecida que as duas doenças apresentam, tornam difícil a distinção entre ambas, fato comprovado em relatos de casos de SARS-CoV-2 que foram tidos como falso positivos para dengue (YAN et al., 2020). A pandemia do COVID-19 tem o potencial de sobrecarregar o Sistema de Saúde e somado aos casos da Dengue pode não haver leitos suficientes para acomodar todos pacientes que precisam ser hospitalizados. 
Os coronavírus são membros da subfamília Coronavirinae, da família Coronaviridae e da ordem Nidovirales, englobando os gêneros Alphacoronavírus, Betacoronavírus, Gammacoronavírus e Deltacoronavírus. Os Alfacoronavírus e Betacoronavírus infectam apenas mamíferos, causando doenças respiratórias em humanos e gastroenterite em animais. Os Gammacoronavírus e Deltacoronavírus infectam aves, apesar de alguns deles também infectarem mamíferos (CUI et al., 2018). Recentemente, foi detectado um coronavírus (SARS-CoV-2) com capacidade de infectar humanos. Ele é um vírus de RNA de fita simples de sentido positivo, pertencente ao gênero Betacoronavírus, com uma identidade de sequência de 88 a $96 \%$ de três coronavírus do tipo SARS, derivados de morcegos (bat-SL-CoVZC45, morcego-SL CoVZXC21, RaTG13) e das cepas de coronavírus isoladas em pangolins (ROSE et al., 2020). Ao se comparar alfa e betacoronavírus, foi identificado que o SARS-CoV-2 parece ser otimizado para ligação ao receptor humano enzima conversora de angiotensina 2 (ECA2) (ANDERSEN et al., 2020). Essa enzima é altamente expressa em células epiteliais alveolares pulmonares e representa um importante portal para a infecção por SARSCoV-2, embora os mecanismos específicos ainda sejam incertos. A expressão de ECA2 é comumente aumentada em algumas doenças crônicas e sua distribuição tecidual possivelmente é diferente entre adultos e crianças, o que pode ser o motivo de haver diferentes gravidades e afecções em diferentes pessoas (ROSE et al., 2020). Biswas e Sukla (2020) relataram que os mapas de gravidade de dengue e COVID-19 não se sobrepõe, isso porque é provável que ocorra, em regiões endêmicas da dengue, uma proteção cruzada para o SARS-CoV-2, pois o este compartilha semelhança antigênica com o DENV e pode reagir com os seus anticorpos específicos. Dessa forma, em pacientes que foram expostos a dengue pode resultar em uma memória imunológica preexistente para o SARS-CoV-2, na forma de anticorpos DENV e células B e T de memória, podendo desencadear um impacto negativo na transmissão, gravidade e patogênese do SARSCoV-2, sugerindo um achatamento na curva de aumento dos casos de COVID-19 em países endêmicos para Dengue. A Dengue é causada por um arbovírus da família Flaviviridae, que também inclui outros vírus relevantes, como o vírus da febre amarela e vírus Zika. Esse Flavivírus possui quatro sorotipos conhecidos $(1,2,3,4)$ e um RNA de cadeia positiva, subunidades funcionais, incluindo três proteínas estruturais (capsídeo, membrana e envelope) e sete proteínas não estruturais (NS1, NS2A/B, NS3, NS4A/B e NS5). A protease NS3 confere um mecanismo catalítico ao vírus da dengue, e por ter um papel essencial em sua replicação é um alvo promissor para desenvolvimento de inibidores terapêuticos para o tratamento de Dengue (LIM et al., 2018). A dengue é endêmica na maioria das regiões tropicais e subtropicais, e os números dos casos costumam aumentar no início do ano devido às estações chuvosas e às altas temperaturas, com picos da infecção entre os meses de março e abril, condições que favorecem a reprodução do mosquito vetor (VON RANDOW et al., 2017). Na análise correlacional reportou que a previsão de pico do SARS-CoV-2 no Brasil deverá ocorrer entre o final do mês de abril e início de maio, quando as doenças respiratórias são mais frequentemente encontradas. Essa coincidência temporal pode 
implicar na emergência de dois surtos concomitantes, desencadeando risco de colapso no Sistema de Saúde do país (LORENZ et al. 2020). Em relação ao modo de transmissão, foi demonstrado que o contato direto de pessoa para pessoa é o fator principal para a propagação de SARS-CoV-2 (BRASIL, 2020). Paralelo a isso, a dengue é a mais prevalente doença viral transmitida por mosquitos, principalmente em regiões tropicais e subtropicais. O DENV-2 atualmente é o sorotipo predominante no país, sendo o mais detectado nas Regiões Centro-Oeste, Sudeste, Sul e Norte, sendo o sorotipo DENV-1, no entanto, o mais predominante na região Nordeste (BRASIL, 2020). A transmissão ocorre pela picada da fêmea do mosquito Aedes aegypti (Diptera: Culicidae), uma espécie sinantrópica e antropofílica com preferência e facilidade COVID-19 no Brasil de proliferação em áreas urbanas densamente povoadas. (CAVALLI et al., 2019). O perfil de acometimento do SARS-CoV-2 é mais prevalente em adultos e em idosos, isso porque a imunosenescência pode exacerbar baixos níveis de imunidade protetora na terceira idade. Além disso, as doenças de coronavírus sazonais (sCovs) foram mais notadas em crianças menores de 5 anos e em idosos, em contrapartida no SARS-CoV-2 poucos são os casos relatados em crianças, isso se deve porque o COVID-19 está relacionado ao CoV-OC43, o SCoV mais predominante em pacientes menores de 5 anos de idade. Dessa forma, é possível que a imunidade cruzada preexistente confira proteção e atenue a gravidade do COVID-19, levando a menos crianças testadas e hospitalizadas

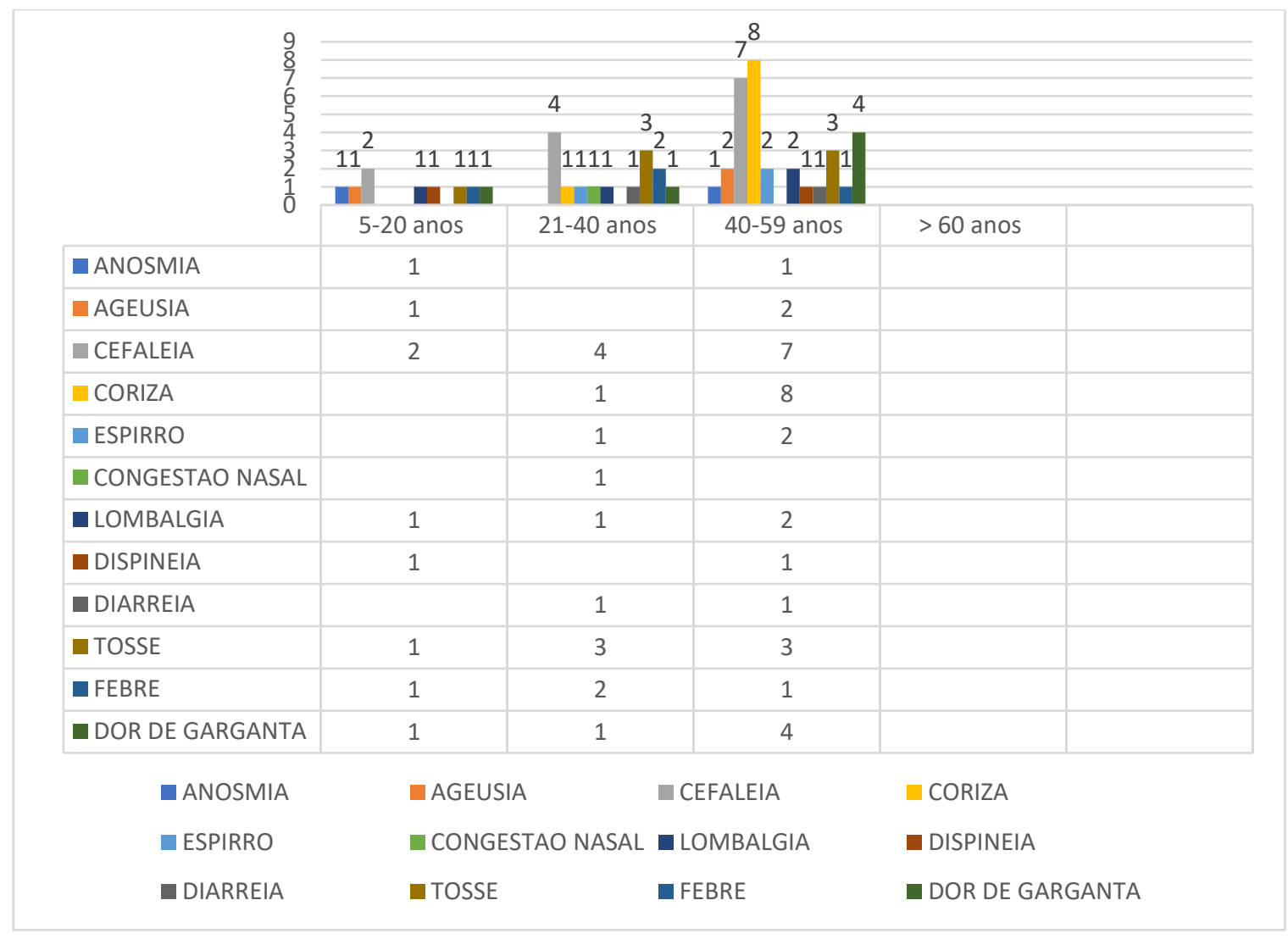


Dentre os sintomas a cefaleia, coriza, lombalgia, diarreia, tosse e dor de garganta são os mais frequentes. Muito dos sintomas podem vim isoladamente ou associados, e assim aumentando o nível de gravidade do Covid-19, e uma doença com essas características é muito difícil de ser parada.

O número de casos vem aumentado, e dentre a população pesquisada foi possível observar que $68 \%$ eram mulheres com idade entre 18 e 59 anos, e os homens com porcentagem de $32 \%$ entre 7 e 55 anos. Pessoas com idade maior a 60 anos não foram notificadas no período da pesquisa tanto do sexo masculino como feminino, ressaltando que a vacina contra a Covid nessa faixa etária já foi realizada.

O Covid- 19 e a dengue há diversos sintomas similares podendo ser confundido quando apresentados isoladamente ou até mesmo em conjunto a depender dos sintomas, dentre os sintomas frequentes em ambas as doenças estão febre, mialgia, cefaleia, dor abdominal, diarreia, diante desse fato e necessário sempre suspeita de ambas as doenças até sua adequada investigação, e assim estipular adequado tratamento. Assim como na dengue o covid- 19 e mais graves em pessoas com a presença de comorbidades e idosos.

Ressaltando que o covid- 19 e uma doença nova e que ainda a muita a ser descoberto, e para tanto e de suma importância a continua monitorização dos sintomas que surgem com o avançar das doenças. E companha a mudanças decorridas após termino por faixa etária da vacinação do covid-19.

Os principais sintomas da dengue clássica (Brasil. Ministério da Saúde- 2002) tem geralmente febre alta, início súbito, cefaleia, mialgia, prostração, artralgia, anorexia, astenia, dor retroorbital, náuseas, vômitos, exantema e prurido cutâneo.

Em comparativo percebemos que os sintomas como febre, cefaleia, mialgia, vômitos, são bem semelhantes entre ambas as doenças, porem sinais de artralgia, dor retroorbital, exantema e prurido são mais característicos da dengue e os sintomas gripais mais característicos do covid-19.

\section{CONSIDERAÇÕES FINAIS}

A população após mais de um ano de pandemia e já a vários anos convivendo com a dengue ainda apresenta surpresa sobre os cuidados básicos de como evitar ambas as doenças, tem resistência ao usar corretamente a máscara, lavagem das mãos, manter o distanciamento e evitar aglomerações, sobre a dengue não tem os devidos cuidados em relação a água parada. Muitos afirmaram que só começou a mudar as atitudes após adoecerem ou alguém próximo.

Notámos que alguns sintomas são parecidos entre dengue e Covid-19, diferenciando principalmente sobre os sintomas gripais que estão ausentes na Dengue. O tempo entre o aparecimento dos sintomas e a busca por ajuda médica é de grande importância para poder diferenciar ambas doenças e focar no tratamento de maneira correta seguindo o protocolo do Ministério da Saúde. 


\section{REFERÊNCIAS}

ANDERSEN, K. G. et al. The proximal origin of SARS-CoV-2. Nature Medicine, [s.1.], v. 26, n. 4, p. 450452, 2020. http://dx.doi.org/10.1038/s41591-020-0820-9

BARRETO ML, Barros AJD, Carvalho MS, Codeço CT, Hallal PRC, Medronho RA. O que é urgente e necessário para subsidiar as políticas de enfrentamento da pandemia de COVID-19 no Brasil? Rev Bras Epidemiol 2020; 23:e200032.

BISWAS, S.; SUKLA, S. COVID-19 virus infection and transmission are observably less in highly Dengue-endemic countries: Can Dengue vaccines be "repurposed" to prevent COVID-19?. Preprints, [s.1.], p. 1-13, 2020. http://dx.doi.org/10.31219/osf.io/dzygw.

BRASIL. Ministério da Saúde. Coronavírus (COVID-19): Medidas não Farmacológicas. 2020. Disponível em: https://coronavirus.saude.gov.br/medidas-nao-farmacologicas BRASIL.

CORONAVÍRUS BRASIL. Síntese de casos, óbitos, incidência e mortalidade. 2020. Disponível em: https://covid.saude.gov.br/._Acesso em: 28 mar. 2021.

COVID-19 NO BRASIL. Predefinição: dados epidemiológicos da Covid-19 no

Brasil. 2020. Disponível em: https://susanalitico.saude.gov.br/extensions/covid19_html/covid19_html.html. Acesso em: 28 mar. 2021

GOVERNO DO ESTADO DE SÃO PAULO. Sobre dengue. 2020. Disponível em: http://portal.saude.sp.gov.br/resources/cve-centro-de-vigilanciaepidemiologica/areas-devigilancia/doencas-de-transmissao-por-vetores-ezoonoses/dengue.htm. Acesso em: 28 mar. 2021. GUZMAN, Maria G; HARRIS, Eva. Dengue. The Lancet, [S.L.], v. 385, n. 9966, p. 453-465, jan. 2015. Elsevier BV. http://dx.doi.org/10.1016/s01406736(14)60572-9.

INSTITUTO FIO CRUZ. Dengue vírus e vetor. 2020. Disponível em: http://www.ioc.fiocruz.br/dengue/textos/longatraje.html\#: :text=Hoje\%2C\%20o

\%20mosquito\%20\%C3\%A9\%20encontrado,\%2D1\%20e\%20DENV\%2D4.LI, F.; SHI, Z. Origin and evolution of pathogenic coronaviruses. Nature Reviews Microbiology, [s.1.], v. 17, n. 3, p. 181-192, 2018. http://dx.doi.org/10.1038/s41579-018-0118-9.

LORENZ, C. et al. COVID-19 and dengue fever: a dangerous combination for the health system in Brazil. Travel Medicine And Infectious Disease, [s.1.], p. 1-2, 2020. http://dx.doi.org/10.1016/j.tmaid.2020.101659 Ministério da Saúde. Saúde de A a Z: Dengue. Disponível em: https://www.saude.gov.br/saude-deaz/dengue BRASIL. Ministério da Saúde. Secretaria de Ciência, Tecnologia, Inovação e Insumos Estratégicos em Saúde.

OPEN DATA SUS. Notificações de síndrome gripal. 2020. Disponível em: https://opendatasus.saude.gov.br/dataset/casos-nacionais. Acesso em: 28 mar. 2021.

ROSE, D. U. de et al. Novel Coronavirus disease (COVID-19) in newborns and infants: what we know so far: what we know so far. Italian Journal of Pediatrics, [s.1.], v. 46, n. 1, p. 1-8, 2020. http://dx.doi.org/10.1186/ s13052-020-0820-x

SAAVEDRA-VELASCO, M. et al. Coinfección entre dengue y COVID-19: necesidad de abordaje en zonas endémicas. : Necesidad de abordaje en zonas endémicas. Revista de La Facultad de Ciencias Médicas de 
Córdoba, [s.1.], v. 77, n. 1, p. 52-54, 2020. Universidad Nacional de Cordoba. http://dx.doi. org/10.31053/1853.0605.v77.n1.28031

VON RANDOW, R. M. et al. Juntos no Controle do Aedes aegypti: Educação em Saúde. JMPHC | Journal of Management \& Primary Health Care | ISSN 2179-6750, v. 7, n. 1, p. 137-137, 2017.

YAN, Gabriel et al. Covert COVID-19 and false-positive dengue serology in Singapore. The Lancet Infectious Diseases, [s.1.], p. 1-1, 2020. http://dx.doi.org/10.1016/s1473-3099(20)30158-4.

Ministério da Saúde (BR) . Secretaria de Ciência, Tecnologia, Inovação e Insumos Estratégicos em Saúde . Acurácia dos testes diagnósticos registrados para a COVID-19: versão 1 [ Internet ]. Brasília : Ministério da Saúde ; 2020 [ citado 2020 jun 1 ]. 19 p. Disponível em: https://www.sbmfc.org.br/wpcontent/uploads/2020/04/Acur_cia_dos_testes_para_COVID_19_1586558625.pdf.pdf

Brasil. Ministério da Saúde. Fundacão Nacional de Saúde. Dengue: aspectos epidemiológicos, diagnóstico e tratamento / Ministério da Saúde, Fundação Nacional de Saúde. - Brasília: Fundação Nacional de Saúde, 2002. 20p.: il. - (Série A. Normas e Manuais Técnicos, $\mathrm{n}^{\circ}$ 176) 